\title{
ANTIBIOTICOPROFILAXIA NA COLECISTECTOMIA VIDEOLAPAROSCÓPICA ELETIVA: ESTUDO PROSPECTIVO RANDOMIZADO E DUPLO CEGO
}

\author{
ANTIBIOTIC PROPHYLAXIS IN ELECTIVE LAPAROSCOPIC CHOLECYSTECTOMY: A \\ PROSPECTIVE, RANDOMIZED AND DOUBLE BLIND STUDY
}

\author{
Hamilton Petry de Souza, TCBC-RS ${ }^{\mathbf{~}}$ Ricardo Breigeiron, TCBC-RS²; \\ Hugo Moreira da Cunha ${ }^{3}$; Eduardo Deves ${ }^{4}$
}

\begin{abstract}
RESUMO: Objetivo: Identificar a necessidade de profilaxia antibiótica em colecistectomias videolaparoscópicas eletivas. Método: Estudo prospectivo, randomizado e duplo-cego, em pacientes submetidos à colecistectomia videolaparoscópica eletiva, durante o período de Junho de 2003 a Julho de 2007, com alocação de 163 pacientes em dois grupos: A (n=82), recebeu profilaxia com cefoxitina $2 \mathrm{~g}$ intravenoso na indução anestésica; B $(\mathrm{n}=81)$, recebeu solução salina 2 mililitros intravenoso. A equipe e a técnica cirúrgica utilizadas foram as mesmas. O desfecho avaliado foram complicações infecciosas de sítio cirúrgico, isto é, infecção de ferida operatória e abscessos superficiais e/ou profundos. Os pacientes foram revisados em sete e 28 dias pós-operatório. Os dados foram analisados pelo Teste exato de Fisher $(\mathrm{p}<0,05)$. Resultados: O presente estudo demonstrou uma taxa de complicações infecciosas de $4,76 \%$, no Grupo A e de $6,17 \%$ no Grupo B. Não houve diferença estatisticamente significativa ( $p=0,746)$ nas taxas de complicações infecciosas. Os grupos foram homogêneos e comparáveis. Conclusão: Pacientes submetidos à colecistectomia videolaparoscópica eletiva, de baixo risco cirúrgico, não necessitam de antibioticoprofilaxia, pois a mesma não traz redução das taxas de infecção (Rev. Col. Bras. Cir. 2008; 35(3): 168-172).
\end{abstract}

Descritores: Colecistectomia laparoscópica; Antibioticoprofilaxia

\section{INTRODUÇÃO}

A colecistectomia vídeolaparoscópica (CVL) tem baixo risco para complicações infecciosas, com média entre $0,4 \%$ a $1,1 \%$ em duas meta-análises recentes ${ }^{1,2}$. Esse resultados são significativamente mais baixos que nas colecistectomias convencionais ${ }^{3}$ mas, mesmo assim, muitos cirurgiões ainda usam antibióticos profiláticos, conduta que varia amplamente ${ }^{4,5}$

A CVL foi descrita na literatura médica pela primeira vez em março de 1987 por Mouret ${ }^{6}$, em Lyon, França, sendo sua técnica aperfeiçoada um ano depois por Dubois ${ }^{7}$. Atualmente, este é um dos procedimentos mais realizados em cirurgia. Somente nos Estados Unidos são realizados, anualmente, cerca de 750.000 procedimentos $^{2}$.

Atualmente, laparoscopia é “padrão-ouro" na colecistectomia ${ }^{1,2}$. As principais vantagens deste procedimento incluem menos dor pós-operatória, estadia hospitalar mais curta, retorno mais rápido à ingesta de alimentos e ao trabalho e diminuição considerável das complicações sépticas perioperatórias ${ }^{8}$. Apesar disso, os mesmos critérios de profilaxia antibiótica aplicados para a cirurgia convencional são rotineiramente usados na cirurgia laparoscópica, sem que haja evidência clara de sua necessidade ${ }^{1-4}$.

Sabe-se que a incidência de infecção de sítio cirúrgico é muito baixa após CVL. A possibilidade de infecção cresce na relação direta com a idade (a maioria dos estudos sinaliza como acima dos 60 anos de idade), infecção ativa (colecistite aguda), presença de obstrução e/ou litíase de via biliar principal, episódios de pancreatite biliar prévios, obesidade e imunossupressão.

As infecções cirúrgicas constituem parcela significativa das infecções em pacientes hospitalizados, correspondendo à cerca de $25 \%{ }^{9-12}$. As bactérias são encontradas em $90 \%$ das incisões cirúrgicas, sendo crescente seu aumento do início ao fim de um procedimento cirúrgico ${ }^{12}$. Estas infecções são causa importante de morbidade e mortalidade no pós-operatório, além de aumentarem os custos hospitalares $^{9,10,13,14}$

Há muitos motivos para justificar a busca pelo paciente que desenvolverá uma infecção do sítio cirúrgico e tentar prevení-la. Infecções da ferida operatória retardam o retorno às atividades laborativas, aumentam o sofrimento e despendem dinheiro, tempo e recursos hospitalares ${ }^{14-18}$.

1. Professor Adjunto e Coordenador do Departamento de Cirurgia da Faculdade de Medicina - Pontifícia Universidade Católica do Rio Grande do Sul (PUCRS); Chefe do Serviço de Cirurgia do Hospital São Lucas da PUCRS (HSL); Fellow American College of Surgeons. Fellow Surgical Infection Society; Doutor em Cirurgia pela UNICAMP.

2. Membro do Serviço de Cirurgia do HSL- Departamento de Cirurgia da Faculdade de Medicina PUCRS; Especialista em Cirurgia do Trauma; Mestre em Cirurgia- PUCRS.

3. Cirurgião Vascular; Ex-Residente do Serviço de Cirurgia Geral / HSL-PUCRS.

4. Residente do Serviço de Cirurgia Geral /HSL-PUCRS.

Recebido em 12/11/2007

Aceito para publicação em 07/02/2008

Conflito de interesses: nenhum

Fonte de financiamento: nenhuma

Trabalho realizado no Serviço de Cirurgia Geral do Hospital São Lucas da Pontifícia Universidade Católica do Rio Grande do Sul. 
Em 1964, O National Research Council (NRC) propôs o uso da classificação de feridas operatórias para predizer o risco de infecção no sítio operatório ${ }^{17}$.

O risco de infecção varia de menos de $1 \%$ nos procedimentos cirúrgicos limpos para mais de $50 \%$ nos procedimentos considerados sujos. A classificação do risco de infecção em graus de contaminação do procedimento cirúrgico não leva em consideração a dificuldade técnica da cirurgia, o estado pré-operatório do paciente e risco anestésico, fatores esses que influem no risco de infecção ${ }^{9,10}$. Mesmo que essa classificação já esteja superada, já que outras mais recentes foram introduzidas, com melhor definição e abrangência ${ }^{17}$, do ponto de vista prático a denominada "classes de Altemeier" continua sendo muito usada. ACVLé classificada como classe II de Altemeier, limpa-contaminada ${ }^{19}$. Portanto, há indicação, em tese, para o uso de antibiótico profilático.

Organismos americanos, como o Centers for Disease Control $(C D C)$, indicam cefalosporina de primeira geração (cefazolina ou cefalotina) como a droga indicada para profilaxia em cirurgia biliar com essas características. Mesmo sendo utilizada em dose única, devido ao volume de cirurgias realizadas anualmente, os custos de seu uso desnecessário podem chegar a valores vultosos. Logo, se sua utilização é dispensável, principalmente se ineficaz e ineficiente, a economia torna-se significativa, além de racionalizar o uso de antibióticos

O objetivo deste trabalho é buscar, através de estudo prospectivo, duplo-cego e randomizado, identificar a necessidade de profilaxia antibiótica em CVLs. Seu foco são pacientes sem fatores de risco para infecção, portanto de baixo risco cirúrgico, em condição eletiva.

\section{MÉTODO}

Estudo prospectivo randomizado e duplo-cego, sendo recrutado um total de 199 pacientes submetidos à CVL eletiva, sendo que, desses, 163 preencheram critérios de inclusão e 36 foram excluídos. Os critérios de exclusão foram: presença de colecistite aguda e/ou coledocolitíase, uso de antibioticoterapia nas 48 horas prévias ao ato cirúrgico e conversão ao método convencional (aberto). Os pacientes foram alocados em dois grupos: $\mathrm{A}(\mathrm{n}=82)$ que recebeu profilaxia com cefoxitina $2 \mathrm{~g}$ intravenoso durante a indução anestésica e B $(n=81)$ que recebeu 2 mililitros de solução salina intravenosa no mesmo período. O uso de cefoxitina deve-se à padroniza- ção, na equipe que realizou o estudo, do Serviço de Cirurgia Geral e do Aparelho Digestivo do HSL/PUCRS, como a droga para profilaxia nos procedimentos que envolvem o trato digestório.

Tanto o cirurgião quanto o paciente não tinham ciência de qual grupo o paciente faria parte (duplo cegamento). A randomização ficou a cargo do anestesiologista da equipe cirúrgica. Os grupos foram comparados quanto à idade, sexo, comorbidades, crises de dor há menos de 30 dias e tempo cirúrgico pelo métodos Qui-Quadrado e Teste exato de Fischer. As comorbidades consideradas foram cardiopatia isquêmica, diabete mellitus, hipertensão arterial sistêmica, imunossupressão, obesidade e hepatites crônicas. O desfecho avaliado foram complicações infecciosas de sítio cirúrgico: infecção de FO e abscessos superficias e/ou profundos. Os pacientes foram reavaliados em sete e 28 dias após a cirurgia. O protocolo de pesquisa foi aprovado pelo Comitê de Ética em Pesquisa da PUCRS. Todos pacientes recebiam e assinavam o Termo de Consentimento Informado.

Números absolutos e percentagens foram utilizados para demonstração dos dados e o teste exato de Fisher para a análise estatística, sendo considerado significativo o nível de confiança de $95 \%(\mathrm{p}<0,05)$.

Complicações infecciosas foram definidas como febre> $38^{\circ}$ ( excluindo $1^{\circ}$ pós-operatório) ou coleção purulenta em sítios cirúrgicos, com ou sem culturais positivos de locais como ferida operatória, trato urinário, respiratório e cavidade abdominal, seguindo definições do $C D C^{20}$. Registro de Ensaios Clínicos: 30-31504 (CEP/PUCRS).

\section{RESULTADOS}

Após coleta de dados através de revisão de prontuários, não foi observado diferença nos grupos ao serem analisados quanto à idade, sexo, comorbidades, crises de dor há menos de 30 dias da cirurgia e tempo cirúrgico médio (tabela 1).

Quanto às complicações infecciosas pós-operatórias, foram constatados quatro eventos no Grupo A e cinco no Grupo B. As complicações ocorridas estão relacionadas na tabela 2.

A análise estatística demonstrou semelhança entre as duas amostras. Não foi encontrada diferença estatisticamente significativa nas taxas de complicações infecciosas $(\mathrm{p}=0,746)$.

Tabela 1 - Estratificação dos grupos.

\begin{tabular}{|c|c|c|c|}
\hline & $\operatorname{Grupo} A(n=82)$ & Grupo B $(n=81)$ & Valor p* \\
\hline Sexo-Feminino $(\%)$ & $(78 \%)$ & $(85 \%)$ & \\
\hline Masculino (\%) & $(22 \%)$ & $(15 \%)$ & 0,240 \\
\hline Idade Média (ANOS) & $45,6 \quad(20-81)$ & $44,8 \quad(19-75)$ & 0,330 \\
\hline Tempo Cirúrgico Médio (MIN) & $112,5 \quad(60-195)$ & $112(60-180)$ & 0,851 \\
\hline Crise de Dor $<30$ & $(41,46 \%)$ & $(36,25 \%)$ & 0,765 \\
\hline \multicolumn{4}{|l|}{ Dias da Cirurgia (\%) } \\
\hline Presença de Comorbidades (\%) & $(40,34 \%)$ & $(38,27 \%)$ & 0,873 \\
\hline
\end{tabular}

\footnotetext{
* Significativo para $p<0,05$.
} 
Tabela 2 - Complicações infeciosas.

\begin{tabular}{|c|c|c|}
\hline & $\operatorname{Grupo} A(n=82)$ & Grupo B $(n=81)$ \\
\hline Infecção FO (\%) & $2 \quad(2,38 \%)$ & $2 \quad(2,47 \%)$ \\
\hline Abscesso Intra-Abdominal (\%) & $2(2,38 \%)$ & $2(2,47 \%)$ \\
\hline Celulite Parede Abdominal (\%) & 0 & $1 \quad(1,23 \%)$ \\
\hline Total $*(\%)$ & $4 \quad(4,76 \%)$ & $5 \quad(6,17 \%)$ \\
\hline
\end{tabular}

*Teste exato de Fisher: $\mathrm{p}=0,746$.

As infecções de FO foram tratadas com medidas locais (drenagem) e antibioticoterapia, no caso da celulite de parede abdominal. Os processos intracavitários foram drenados através de punção orientada por método de imagem (Ecografia) e antibioticoterapia. Não houve mortalidade relacionada ao estudo. Não foram observadas reações adversas à cefoxitina.

\section{DISCUSSÃO}

A antibioticoprofilaxia em CVL eletiva é assunto de extensa controvérsia na medicina. Apesar de diversos trabalhos bem estruturados ${ }^{3,4,5,8,21-24}$ e metanálises recentes ${ }^{, 25,26}$ comprovarem que ela não diminui o risco de complicações infecciosas, diversos cirurgiões ainda a utilizam. Em pacientes selecionados, de baixo risco, sem evidência de inflamação aguda, exploração de via biliar ou coledocolitíase, que não tenham apresentado pancreatite biliar ou serem imunocomprometidos, qual seria a razão para usá-la?

A profilaxia antibiótica tem mostrado seu valor na diminuição da mortalidade e morbidade nos últimos 35 $\operatorname{anos}^{12,14,16}$. Embora reconhecida sua relevância, há controvérsias quanto ao seu modo de uso. O antimicrobiano ideal não deve induzir resistência bacteriana, deve ter penetração tecidual efetiva, baixa toxicidade, além de não interferir com drogas anestésicas e, sobretudo, ser de baixo custo ${ }^{12,14}$. Nenhum agente disponível perfaz todos estes requisitos. A escolha, em cirurgias do aparelho digestório, recai, via de regra, em droga(s) eficaz(es) contra germes gram- negativos aeróbios e anaeróbios ${ }^{12}$

A profilaxia antimicrobiana não é realizada para erradicar completamente os microorganismos do tecido, mas para reduzir o número de microorganismos a tal extensão que os mecanismos de defesa do hospedeiro sejam hábeis para efetivamente prevenir a infecção pelos microorganismos contaminantes ${ }^{14}$.

Recomenda-se que o antibiótico seja iniciado na indução anestésica e repetido conforme sua meia-vida e farmacodinâmica, sendo encerrada ao final da cirurgia ou no pós-operatório por um período máximo de 24 horas $^{14}$. A profilaxia realizada incorretamente ou por períodos prolongados, com início, dose ou drogas inadequadas, aumenta o risco de infecção em até $38 \%$.

A profilaxia antibiótica está indicada em procedimento cirúrgico que apresente potencial de complicação infecciosa ou em procedimentos em que a infecção não é comum, porém com sérias ameaças ou complicações com risco de vida ${ }^{9,12}$.
Análises de custos do emprego de antibióticos na profilaxia operatória revelaram vantagens imediatas no uso adequado, que significou, especialmente, redução da infecção de sítio cirúrgico e do tempo de permanência hospitalar, além dos custos inerentes à investigação e ao tratamento dessas complicações infecciosas ${ }^{12}$. Existem vários esquemas profiláticos possíveis, não sendo definido se uma combinação de drogas, droga única ou qual droga seja o melhor esquema $^{12}$.

Durante a era pré-laparoscópica (antes de 1960), infecção após colecistectomia eletiva por laparotomia em pacientes de baixo risco (com ausência de imunossupressão, colecistite aguda ou obstrução / litíase do ducto biliar comum) era infreqüente, com incidência entre zero e $4 \%$. cultura de bile positiva estar associada a altas taxas de infecção ferida operatória (FO), a bactéria isolada da bile e a da infecção da FO são freqüentemente diferentes, sugerindo que a bile não seja o causador do processo infeccioso e, sim, a flora da pele $\mathrm{e}^{10,11}$. Uma análise de $1518 \mathrm{CVL}$ realizadas logo após a introdução do procedimento reportou taxa de infecção de $1,1 \%$, a maioria das quais ( 14 de 16$)$ foram infecções superficiais do portal umbilical. Somente dois abscessos abdominais ocorreram nestes 1518 pacientes $^{4}$.

Tocchi et $a l^{22}$ sugeriram que a profilaxia antibiótica pode ser justificada em CVL eletiva em pacientes com crises de dor há menos de 30 dias da operação e diabéticos. Em sua meta-análise, McGuckin et $a l^{1}$, afirmaram que a profilaxia antibiótica somente deveria ser usada em pacientes de alto risco.

Higgins $e ~_{a l}{ }^{3}$ não encontraram redução nas taxas de infecção ao uso de dose única de cefazolina na indução anestésica em pacientes submetidos à CVL eletiva. Illig et $\mathrm{l}^{8}$ publicaram resultados semelhantes em um estudo comparando três doses pré-operatórias de cefazolina versus placebo em CVL eletivas.

Koc et $a l^{21}$ afirmaram não existir correlação entre a microbiologia biliar e as complicações infecciosas pós-operatórias.

Existe debate permanente sobre a real condição de se executar estudos com metodologia correta em Cirurgia, em especial ensaios clínicos randomizados e prospectivos. A possibilidade de cegamento e, idealmente, duplo cegamento, é quase inviável, ainda que, em casos como o presente estudo, a pesquisa sobre efetividade e comparação de ação de medicamentos, torne essa tarefa factível. Mesmo assim, há inúmeros vieses, o que suscita dúvidas quanto à validade de diversos trabalhos.

Embora não apresente número expressivo de casos, o estudo tem o mérito de ser "duplo cego", fato que o credencia 
a ser apreciado. Como o índice de infecção em sítio cirúrgico em CVL eletiva é baixo ${ }^{1,2,3}$, exige um "n" significativo para evitar conclusão estatística equivocada.

Chang et $a l^{5}$, em trabalho recentemente publicado nessa linha, apresentam uma amostra pouco maior (277 pacientes), sem ser, entretanto, duplo cego .

Apesar do número superior de complicações infecciosas relatadas no Grupo B (placebo), não foi encontrado significância estatística. Os grupos demonstraram-se homogêneos e, portanto, perfeitamente comparáveis, tornando o trabalho reprodutível.
Em conclusão, verifica-se, nesse estudo, que não há razão ou justificativa para a utilização de antibioticoprofilaxia em CVL eletivas em paciente de baixo risco, isto é, sem processos de agudização recentes, obstrução e/ou litíase de via biliar. Somando-se a isso, há o fato que o uso indiscriminado de antibioticoprofilaxia pode induzir resistência, alteração na flora biliar normal e aumentar o número de infecções nosocomiais ${ }^{21}$.

Novos e adequados trabalhos, com número maior de pacientes, são necessários para a confirmação desta conclusão. Enquanto isso, espera-se que o presente possa ter contribuído para o esclarecimento de tão importante questão.

\begin{abstract}
Background: To identify the need for antibiotic prophylaxis usage in routine laparoscopic cholecystectomy. Methods: A prospective, randomized double-blind study was done in patients submitted to routine laparoscopic cholecystectomy from June 2003 to July 2007, with 163 patients divided in two groups: Group $A(n=82)$ received antibiotic prophylaxis with Cefoxitin $2 g$ IV at anesthesia induction; Group B $(n=81)$ received $2 \mathrm{~mL}$ of isotonic sodium chloride solution at same time. Surgical technique and team were the same. The purpose of this study was to search the outcome for surgical site infections and superficial or deep abscesses. The patients were examined at 7 and 28 days after surgery. Data were analyzed by Fisher's exact test. Results: This study showed infection complication rates of $4.76 \%$ in group $A$ and $6.17 \%$ in group B. There were no statistical significant differences $(p=0.746)$ for infection complication rates in both groups. The groups were homogeneous and comparable. Conclusions: Patients submitted to routine laparoscopic cholecystectomy with low surgical risk do not need antibiotic prophylaxis, because it will not result in lower infection rates.
\end{abstract}

Key words: Cholecystectomy, laparoscopy; Antibiotic prophylaxis

\section{REFERÊNCIAS}

1. McGuckin M, Shea JA, Schwartz JS. Infection and antimicrobial use in laparoscopic cholecystectomy. Infect Control Hosp Epidemiol. 1999; 20(9):624-6.

2. Shea JA, Healey MJ, Berlin JA, Clarke JR, Malet PF, Staroscik RN, Schwartz JS, Williams SV. Mortality and complications associated with laparoscopic cholecystectomy. A meta-analysis. Ann Surg. 1996; 224(5):609-20.

3. Higgins A, London J, Charland S, Ratzer E, Clark J, Haun W, Maher DP. Prophylatic antibiotics for elective laparoscopic cholecystectomy: are they necessary? Arch Surg. 1999; 134(6):611-3; discussion 614.

4. Shindholimath VV, Seenu V, Parshad R, Chaudhry R, Kumar A. Factors influencing wound infection following laparoscopic cholescytectomy. Trop Gastroenterol. 2003; 24(2):90-2.

5. Chang WT, Lee KT, Chuang SC, Wang SN, Kuo KK, Chen JS, Sheen PC. The impact of prophylactic antibiotics on postoperative infection complication in elective laparoscopic cholecystectomy: a prospective randomized study. Am J Surg. 2006; 191(6):721-5.

6. Mouret P. From the first laparoscopic cholecystectomy to the frontiers of laparoscopic surgery; the futures prospectives. Dig Surg. 1991; 8:124-5.

7. Dubois F, Berthelot G, Levard H. Laparoscopic cholecystectomy: historic perspective and personal experience. Surg Laparosc Endosc. 1991; 1(1):52-7.

8. Illig KA, Schmidt E, Cavanaugh J, Krusch D, Sax HC. Are prophylactic antibiotics required for elective laparoscopic cholecystectomy? J Am Coll Surg. 1997; 184(4):353-7.
9. Lízan-García M, García-Caballero J,Ansensio-Vegas A. Risk factors for surgical-wound infection in general surgery: a prospective study. Infect Control Hosp Epidemiol. 1997; 18(5):310-5.

10. Holzheimer RG, Haupt W, Thiede A, Schwarzkopf A. The challenge of postoperative infections: does the surgeon make a difference? Infect Control Hosp. Epidemiol.1997; 18(6):449-56.

11. Knight R, Charbonneau P, Ratzer E, Zeren F, Haun W, Clark J. Prophylactic antibiotics are not indicated in clean general surgery cases. Am J Surg. 2001; 182(6):682-6.

12. Schilling J, Michalopoulos A, Geroulanos S. Antibiotic prophylaxis in gastroduodenal surgery. Hepatogastroenterology. 1997; 44(13):116-20.

13. Souza HP, Breigeiron R, Vilhordo DW. Infecção em cirurgia. In: Cavazzola LT, editor. Condutas em cirurgia geral. Porto Alegre: Artmed; 2008. p.50-7.

14. Silver A, Eichorn A, Kral J, Pickett G, Barie P, Pryor V, Dearie MB. Timeliness and use of antibiotic prophylaxis in selected inpatient surgical procedures. The Antibiotic Prophylaxis Study Group. Am J Surg. 1996; 171(6): 548-52.

15. Ferraz AAB, Leão CS, Campos JM, Martins Filho E, Albuquerque AC, Ferraz EM. Profilaxia antibiótica na cirurgia bariátrica: ampicilina/sulbactam x ceftriaxona x ertapenem. Rev Col Bras Cir. 2007; 34(2):73-7.

16. Velasco E, Thuler LS, Martins CAS, Dias LMC, Gonçalvez VM. Profilaxia antimicrobiana em cirurgias oncológicas. Rev Hosp Clin Fac Med Univ São Paulo. 1997; 52 (4):209-16.

17. Nichols RL. Surgical infections: prevention and treatment-1965 to 995 . Am J Surg. 1996; 17(1):68-74.

18. Souza HP, Breigeiron R, Pandolfo G. Antibioticoterapia na sepse abdominal. In: Rasslan S. editor. O doente cirúrgico na UTICBMI. São Paulo: Atheneu; 2001. p. 169. 
19. Altemeier WA, Burke JF, Pruitt BA, Sandusky WR, editors. Manual on control of infection in surgical patients. $2^{\text {nd }}$ ed. Philadelphia: Lippincott; 1984.

20. Horan TC, Gaynes RP, Martone WJ, Jarvis WR, Emori TG. CDC definitions of nosocomial surgical site infections, 1992: a modification of CDC definitions of surgical wound infections. Infect Control Hosp Epidemiol.1992; 13(10):606-8.

21. Koc M, Zulfikaroglu B, Kece C, Ozalp N. A prospective randomized study of prophylactic antibiotics in elective laparoscopic cholecystectomy. Surg Endosc. 2003; 17(11):17168. Epub 2003 Jun 17.

22. Tocchi A, Lepre L, Costa G, Liotta G, Mazzoni G, Maggiolini F. The need for antibiotic prophylaxis in elective laparoscopic cholecystectomy: a prospective randomized study. Arch Surg. 2000; 135(1):67-70; discussion 70.

23. Catarci M, Mancini S, Gentileschi P, Camplone C, Sileri P, Grassi GB. Antibiotic prophylaxis in elective laparoscopic cholescystectomy. Lack of need or lack of evidence? Surg Endosc. 2004; 18(4):638-41. Epub 2004 Feb 2.

24. Orozco H, Mercado MA. The need for antibiotic prophylaxis in elective laparoscopic cholecystectomy [discussion]. Arch Surg. 2000; 135(1):70.
25. Al-Ghnainiem R, Benjamin IS, Patel AG. Meta-analysis suggests antibiotic prophylaxis is not warranted in low-risk patients undergoing laparoscopic cholecystectomy. Br J Surg. 2003; 90(3):365-6.

26. Sanabria A, Valdivieso E, Gomez G. Antibiotic prophylaxis for patients undergoing elective laparoscopic cholecystectomy. (Protocol) Cochrane Database of Systematic Reviews 2007, Issue 4. Art. No.: CD005265.

Como citar este artigo:

Souza HP, Breigeiron R, Cunha HM, Deves E. Antibioticoprofilaxia na colecistectomia videolaparoscópica eletiva: estudo prospectivo randomizado e duplo cego. Rev Col Bras Cir. [periódico na Internet] 2008; 35(3). Disponível em URL: http://www.scielo.br/rcbc

Endereço para correspondência:

Hamilton Petry de Souza

R.Antonio Parreiras,145/apto. 401

90450-050 - Porto Alegre - RS

(51)3336-7505

hpetrys@terra.com.br 